\title{
The long-term effects of splenectomy and subsequent interferon therapy in patients with $\mathrm{HCV}$-related liver cirrhosis
}

\author{
YUJI INAGAKI ${ }^{1}$, KAZUSHI SUGIMOTO ${ }^{1}$, KATSUYA SHIRAKI ${ }^{1}$, MASAHIKO TAMEDA ${ }^{1}$, SATOKO KUSAGAWA ${ }^{1}$, \\ KEIICHIRO NOJIRI $^{1}$, SUGURU OGURA ${ }^{1}$, NORIHIKO YAMAMOTO ${ }^{1}$, YOSHIYUKI TAKEI $^{1}$, \\ MASAAKI ITO ${ }^{1}$, SHUGO MIZUNO ${ }^{2}$, MASANOBU USUI ${ }^{2}$, HIROYUKI SAKURAI ${ }^{2}$ and SHUJI ISAJI ${ }^{2}$ \\ Departments of ${ }^{1}$ Internal Medicine and ${ }^{2}$ Hepatobiliary Pancreatic and Transplant Surgery, \\ Mie University Graduate School of Medicine, Tsu, Mie 514-8507, Japan
}

Received July 9, 2013; Accepted November 11, 2013

DOI: $10.3892 / \mathrm{mmr} .2013 .1856$

\begin{abstract}
Partial splenic embolization (PSE) or splenectomy is widely performed to increase platelet counts for interferon (IFN) therapy. The aim of the present study was to evaluate the long-term effects of splenectomy and subsequent IFN therapy in patients with hepatitis $\mathrm{C}$ virus (HCV)-related liver cirrhosis (LC). The present study included 19 patients with HCV-related LC who underwent splenectomy for thrombocytopenia caused by hypersplenism. IFN therapy was performed in all 19 patients. The effects of splenectomy and subsequent IFN therapy on peripheral blood counts, liver function, carcinogenesis and survival rates were evaluated. Splenectomy was safely performed in all patients without major complications with the exception of portal thrombosis, which, however, it did not affect liver function when treated appropriately. Thrombocytopenia improved and IFN therapy could be performed in all the patients. A sustained virological response (SVR) was not observed in patients with genotype 1 although it was observed in $75 \%$ of patients with genotype 2 . Due to severe side effects, five patients did not undergo scheduled IFN therapy. Over 5 years, the mean platelet number increased from $5.2 \times 10^{4}$ to $16.8 \times 10^{4} / \mathrm{mm}^{3}(\mathrm{P}<0.01)$ and liver function improved following splenectomy (albumin, Alb: 3.5-3.8 g/dl; total bilirubin, T-Bil: $1.0-0.7 \mathrm{mg} / \mathrm{dl}$; prothrombin time, PT: 74.1-97.7\%; total cholesterol; T-cho: $140-168 \mathrm{mg} / \mathrm{dl}$; $\mathrm{P}<0.05$ ). Hepatocellular carcinoma (HCC) occurred in only one patient during long-term observation and follow-up of the patients not presenting with HCC at entry. The results of the present study demonstrate that splenectomy followed by inferon therapy could be beneficial in patients with HCV-related LC.
\end{abstract}

Correspondence to: Dr Katsuya Shiraki, Department of Internal Medicine, Mie University School of Medicine, 2-174 Edobashi, Tsu, Mie 514-8507, Japan

E-mail: katsuyas@clin.medic.mie-u.ac.jp

Key words: HCV, liver cirrhosis, interferon, splenectomy

\section{Introduction}

Chronic hepatitis $\mathrm{C}$ with cirrhosis is a major risk factor for hepatocellular carcinoma (HCC). It has been reported that the annual incidence of $\mathrm{HCC}$ is $\sim 7 \%$ in patients with hepatitis $\mathrm{C}$ virus (HCV)-related liver cirrhosis (LC) (1). In patients with HCV-related LC, achievement of a sustained virological response (SVR) following interferon (IFN) therapy was found to be associated with a reduction in liver-related mortality and with a lower risk of liver-related complications and the occurrence of HCC (2-4). IFN therapy has also been reported to reduce the severity of liver tissue fibrosis and inflammation (5). The patients achieving SVR had the greatest improvements in fibrosis and inflammation. The patients who achieved HCV RNA suppression under the level of detection during treatment, but who relapsed subsequent to treatment discontinuation, also experienced improvements in fibrosis and inflammation.

Pancytopenia is a common manifestation in cirrhotic patients due to the increasing platelet pool in the enlarged spleen, reduction of thrombopoietin production in the failing liver and platelet destruction due to an immunological mechanism. The presence of severe thrombocytopenia in patients with cirrhosis associated with HCV infection limits the use of IFN therapy (6-8). Therefore, management of thrombocytopenia prior to antiviral treatment should be considered.

Laparoscopic splenectomy $(9,10)$, partial splenic embolization (PSE) $(11,12)$ and administration of a thrombopoietin receptor agonist (13) are considered useful treatment options for thrombocytopenia with chronic liver disease. The results of antiviral therapy with thrombopoietin receptor agonists remain under investigation. Splenectomy and PSE could be useful treatments for thrombocytopenia, however, further investigation is required to identify which treatment is better in terms of safety and a successful outcome following antiviral treatment. Splenectomy has a greater effect in raising the platelet count and has recently been employed as a salvage therapy following PSE (14), making it highly reliable for raising platelet counts. However, splenectomy in general is considered to be associated with a potential risk of infection. A meta-analysis of follow-up studies involving 19,680 patients who underwent splenectomy demonstrated that the incidence of 
sepsis among post-splenectomy adult patients was low and that a high mortality rate was observed only among children (15). It appears that splenectomy is reasonably safe in adult patients. We previously reported that splenectomy improves liver function in patients with LC (16). Thus, splenectomy appears to be the definitive treatment for hypersplenism and IFN therapy following splenectomy is efficacious and safe (17-20).

However, there are only a few studies that have examined the long-term effects of splenectomy and subsequent IFN therapy in patients with $\mathrm{HCV}$ cirrhosis and thrombocytopenia. In the present study, the effect of IFN therapy following splenectomy was investigated in patients with HCV-related LC during long-term follow-up.

\section{Materials and methods}

Patients. Between January 2005 and April 2010, 19 HCV-related cirrhotic patients with hypersplenism underwent splenectomy and were followed up for over 2 years at the Mie University School of Medicine (Tsu, Mie, Japan). Patients were diagnosed as having LC using a combination of laboratory tests, including ultrasonography (US) and computed tomography (CT), and this was confirmed during surgery. The eligibility criteria for the present study were adequate liver function (Child-Pugh class A or B) and thrombocytopenia (platelet count $\leq 8 \times 10^{4} / \mathrm{mm}^{3}$ ). The reasons for splenectomy included difficulties in starting or continuing IFN therapy due to thrombocytopenia. The clinical characteristics of the patients prior to splenectomy are shown in Table I. There were 13 patients who did not have HCC, however, six patients had a history of HCC treatment and had received therapy for HCC prior to starting IFN.

Splenectomy. Splenectomy was principally performed laparoscopically, however, whether open surgery or laparoscopy was selected depended on the size of the spleen and the presence of complications. The patient was placed in the right semi-decubitus position under general anesthesia and pneumoperitoneum was induced. Dissection of the splenocolic ligament and parietal peritoneum using the vessel sealing system (Ligasure Atlas ${ }^{\mathrm{TM}}$; Valleylab, Boulder, CO, USA) was started from the lower pole and then proceeded to the upper pole through the lateral side of the spleen. Following sufficient mobilization of the spleen, the splenic hilum was stapled with linear staplers and the fractured spleen was delivered from the extended wound.

Therapy for $H C V$. When platelet counts were increased and the general condition of the patient had stabilized following splenectomy, IFN therapy was initiated. The combination of peginterferon $\alpha-2 b$ (PEG-IFN $\alpha 2 b$; PegIntron; Schering-Plough Pharmaceutical, Osaka, Japan) and ribavirin (RBV; Rebetol; Schering-Plough Pharmaceutical) was used. The dosage was determined using a weight-based regimen. The treatment durations were 48 and 24 weeks for patients with HCV genotypes 1 and 2, respectively. Dose reduction and discontinuation measures were used in the event of cytopenia, other complications and treatment ineffectiveness.

The effect of IFN therapy was classified according to the elimination of HCV-RNA and the alanine aminotransferase
(ALT) value 6 months following the end of treatment. SVR was defined as the persistent disappearance of HCV RNA following therapy, the biochemical response (BR) was defined as normal ALT values without elimination of HCV RNA for at least 6 months following therapy and no response (NR) was defined as persistently elevated or transiently normalized ALT levels without the loss of HCV RNA.

For patients who did not attain SVR, the combination therapy of PEG-IFN $\alpha 2 \mathrm{a}+\mathrm{RBV}$ or IFN $\beta+\mathrm{RBV}$ was used as a follow-up treatment aimed at achieving SVR. If this was not possible due to problems in the past treatment history or blood cell counts, IFN monotherapy was performed wherever possible (specifically, $90 \mu \mathrm{g}$ of PEG-IFN $\alpha 2 \mathrm{a}$ or $180 \mu \mathrm{g}$ of PEG-IFN $\alpha 2$ a was used every 1 or 2 weeks or 3,000,000 units of natural IFN was used three times a week).

Follow-up of patients. Patients were followed up on a monthly basis following the diagnosis of cirrhosis by monitoring hematological, biochemical and virological data. Imaging studies were conducted three or more times per year in the majority of patients using CT or US. Angiography was performed only when there was a high suspicion of HCC on CT or US.

The follow-up period was defined as the duration between the date of splenectomy and either the date of mortality or the latest date of confirmed survival.

Statistical analysis. Data values are expressed as the means \pm standard deviation. Pre-splenectomy and 5 years post-splenectomy data were compared using a paired t-test. $\mathrm{P}<0.05$ was considered to indicate a statistically significant difference.

\section{Results}

Splenectomy. Splenectomy was safely performed in all the patients without major complications with the exception of portal thrombosis, which occurred in several patients, but did not affect liver function when treated appropriately. Significant increases in platelet counts and leukocyte counts were observed following splenectomy. The mean platelet count at 4 weeks following splenectomy was $20.3 \times 10^{4} / \mathrm{mm}^{3}$ $(\mathrm{P}<0.0001)$. The mean leukocyte count at 4 weeks following splenectomy was $6.3 \times 10^{3} / \mathrm{mm}^{3}(\mathrm{P}<0.0001)$. The mean hemoglobin $(\mathrm{Hb})$ prior to and following splenectomy remained almost identical at $12.1 \mathrm{~g} / \mathrm{dl}$ prior to and $11.7 \mathrm{~g} / \mathrm{dl}$ following (not significant; Fig. 1).

Response to IFN therapy. IFN therapy was initiated in all patients. Table II shows the effect of IFN therapy following splenectomy. SVR was not observed in patients with genotype 1 , but was observed in $75 \%$ of patients with genotype 2 . Out of 15 patients with genotype 1, five discontinued IFN therapy as they exhibited certain side effects, including interstitial pneumonitis, $\mathrm{HCV}$-associated glomerulonephritis or leukopenia. All the patients with genotype 2 were able to continue IFN therapy.

In the group not achieving SVR, a switch to PEG-IFN $\alpha 2 \mathrm{a}+$ $\mathrm{RBV}$ or to long-term IFN monotherapy was considered to achieve SVR. In the group with no previous HCC, 4 patients received $90 \mu \mathrm{g}$ of PEGIFNa2a every other week, 1 patient 
Table I. Clinical features of hepatitis C patients prior to splenectomy.

\begin{tabular}{lc}
\hline Factor $(\mathrm{n}=19)$ & Values \\
\hline Age (years) & $56 \pm 7$ \\
Male/female & $11 / 8$ \\
Child-Pugh score $(5 / 6 / 7 / 8)$ & $6 / 10 / 2 / 1$ \\
Serum albumin $(\mathrm{g} / \mathrm{dl})$ & $3.5 \pm 0.5$ \\
Total bilirubin $(\mathrm{mg} / \mathrm{dl})$ & $1.0 \pm 0.4$ \\
Prothrombin time $(\%)$ & $74.1 \pm 12.1$ \\
WBC count $\left(/ \mathrm{mm}^{3}\right)$ & $2945 \pm 841$ \\
Platelet count $\left(/ 10^{4} \mathrm{~mm}^{3}\right)$ & $5.2 \pm 1.5$ \\
Treatment history for hepatocellular carcinoma & $6 / 13$ \\
Treatment history with interferon & $6 / 13$ \\
HCV genotype $(1 / 2)$ & $15 / 4$ \\
HCV load (log IU/ml) & $5.9 \pm 0.8$
\end{tabular}

WBC, white blood cell; HCV, hepatitis C virus.

Table II. IFN therapy following splenectomy.

\begin{tabular}{lcc}
\hline Factor & $\begin{array}{c}\text { Genotype } 1 \\
(\mathrm{n}=15)\end{array}$ & $\begin{array}{c}\text { Genotype } 2 \\
(\mathrm{n}=4)\end{array}$ \\
\hline Continued & 10 & 4 \\
Discontinued & 5 & 0 \\
SVR & 0 & 3 \\
Relapse & 1 & 1 \\
BR & 2 & 0 \\
NR & 9 & 0
\end{tabular}

IFN, interferon; SVR, sustained virological response; BR, biochemical response; NR, no response.

received $180 \mu \mathrm{g}$ of PEG-IFNa2a weekly, 2 patients received IFN $\beta+$ RBV cotherapy, 2 patients received PEG-IFN $\alpha 2 \mathrm{a}+\mathrm{RBV}$ cotherapy and 2 patients received 3,000,000 units of natural IFN $\alpha$ three times a week. In the group with previous HCC, only 1 patient received PegIFNa2a + RBV cotherapy. IFN could not be used in the other patients due to $\mathrm{HCC}$ recurrence.

Changes in hematological and biochemical parameters following splenectomy. The mean leukocyte and platelet counts increased in patients following splenectomy (Fig. 1). Over 5 years, the mean platelet number increased from $5.2 \times 10^{4}$ to $16.8 \times 10^{4} / \mathrm{mm}^{3}(\mathrm{P}<0.01)$ and the mean leukocyte number increased from $2.9 \times 10^{3}$ to $6.3 \times 10^{3} / \mathrm{mm}^{3}(\mathrm{P}<0.001)$. The mean hemoglobin was virtually unchanged, from 12.1 to $12.8 \mathrm{~g} / \mathrm{dl}$ (not significant). The levels of albumin (Alb), total cholesterol (T-cho), total bilirubin (T-Bil) and prothrombin time (PT) improved following splenectomy. The mean Alb demonstrated an upward trend from 3.5 to $3.8 \mathrm{~g} / \mathrm{dl}$, the mean T-Bil demonstrated a downward trend from 1.0 to $0.7 \mathrm{mg} / \mathrm{dl}$, the mean PT demonstrated an upward trend from 74.1 to $97.7 \%$ and the mean
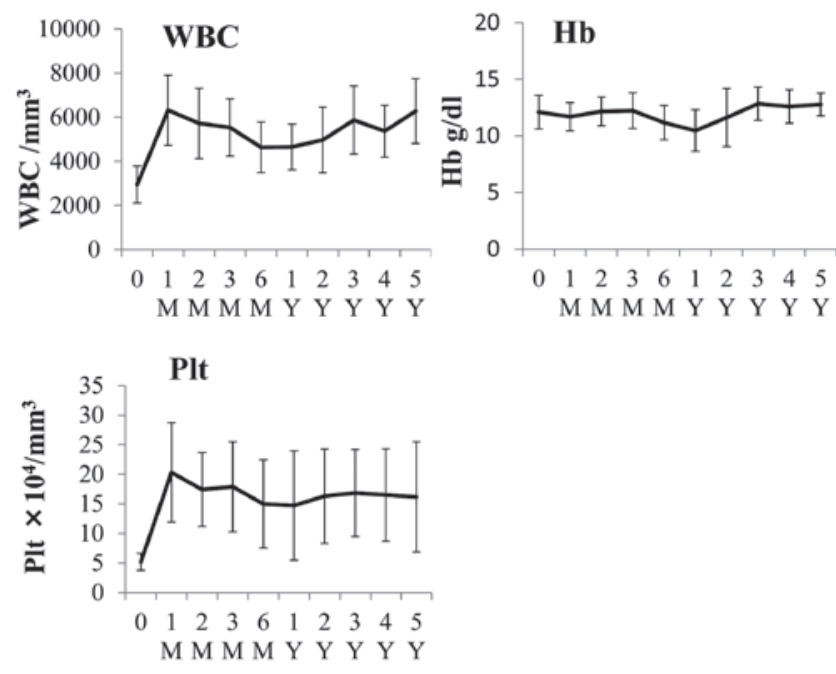

0 , before splenectomy; M, months; Y, years

Figure 1. Changes in peripheral blood counts following splenectomy. WBC, white blood cell; Hb, hemoglobin; Plt, platelets.
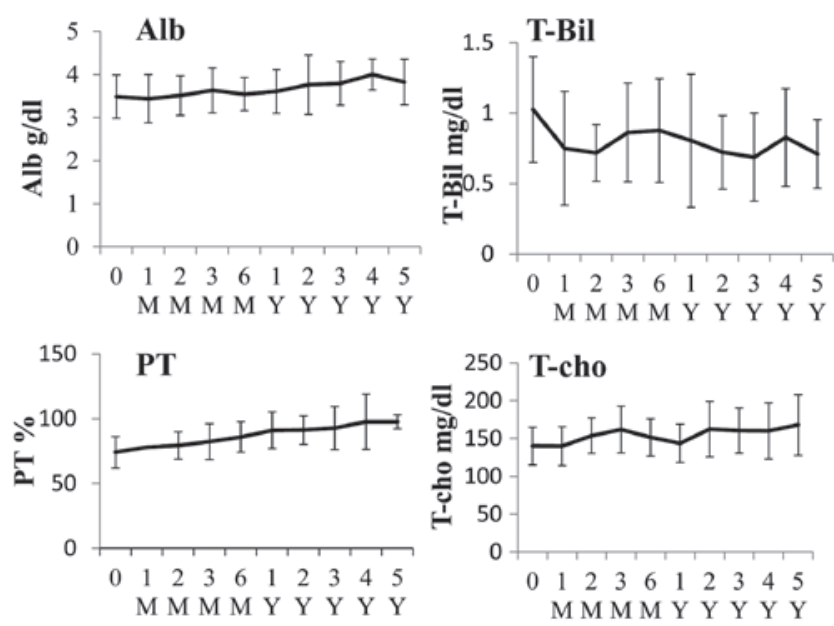

0, before splenectomy; M, months; Y, years

Figure 2. Changes in liver function following splenectomy. Alb, albumin; T-cho, total cholesterol; T-Bil, total bilirubin; PT, prothrombin time.

T-cho increased from 140 to $168 \mathrm{mg} / \mathrm{dl}(\mathrm{P}=0.0421), 5$ years following splenectomy (Fig. 2). These results indicated that, at the very least, there was no deterioration in liver function.

Hepatocarcinogenesis and mortality following splenectomy and IFN combination therapy. Fig. 3 shows the clinical course of the 13 patients without previous HCC. The mean follow-up period in the group without HCC was 4.8 years. Wherever possible, IFN therapy was also administered to patients who did not achieve SVR. New HCC occurred in only one patient during the observation period and all patients survived during this period.

The mean follow-up period was 4.8 years in the group with a history of HCC treatment. HCC recurrence was observed in four patients, however, it was treatable in all the patients. Curative treatment by radiofrequency ablation (RFA) with 


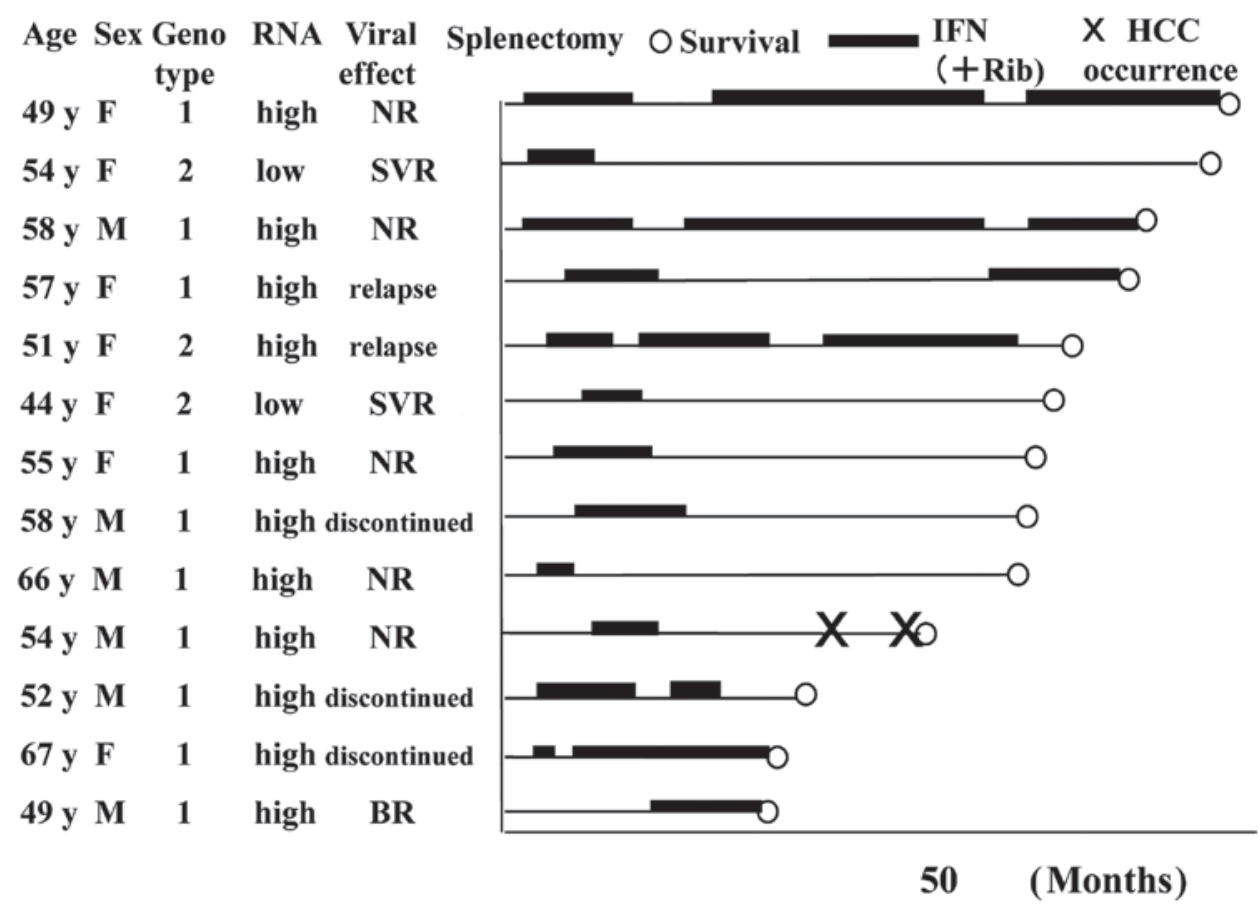

Figure 3. Clinical course following splenectomy in individual patients who underwent IFN therapy. IFN, interferon; HCC, hepatocellular carcinoma; SVR, sustained virological response; BR, biochemical response; NR, no response; Rib, ribavirin.

transcatheter arterial chemoembolization (TACE) in two cases was possible in all cases of HCC recurrence if it was the first recurrence. Three patients had a second recurrence, two of whom were treated by TACE + RFA and one was relieved by partial resection following TACE treatment. One patient had a third recurrence and developed distant metastasis, for which treatment with sorafenib was initiated and TACE + RFA was performed on the liver. There were no cases of liver failure and all patients were Child-Pugh class A for liver fuction at the final follow-up. All patients survived the follow-up period.

\section{Discussion}

In the present study, HCV-related cirrhotic patients with hypersplenism underwent splenectomy to facilitate the initiation and completion of IFN therapy. Since LC can cause life-threatening conditions, including liver failure and HCC within a few years, it is evident that treatment of such a severe disease is an important medical objective. Although elusive, SVR in patients with chronic hepatitis $C$ and advanced liver disease remains a significant objective. Previous studies $(2,5)$ have demonstrated that in patients receiving treatment, particularly those whose virus is permanently eradicated, the progression of fibrosis may be arrested and the cirrhosis reversed. Despite the less favorable histological benefit in non-responder patients, certain patients also demonstrated a reduction in the histological activity index and fibrosis (5). In addition, evidence suggests that antiviral therapy decreases the rates of liver-related complications and mortality.

The present cases had severe leukopenia and thrombocytopenia. Significant increases in leukocyte and platelet counts were observed following splenectomy. These results are consistent with those obtained in other studies (9,10,17-20). Steady improvement of leukocyte and platelet counts is beneficial for subsequent antiviral treatment. PSE can be an effective treatment for thrombocytopenia with hypersplenism. Splenectomy and PSE could be useful treatments for thrombocytopenia, however, further investigation is required to identify which treatment is better in terms of safety and a successful outcome following antiviral treatment. In cases of severe thrombocytopenia, we hypothesize that splenectomy is more beneficial from the perspective of increasing platelet counts for antiviral therapy. In cirrhotic patients with severe thrombocytopenia, splenectomy may be a better supportive intervention than PSE (20). Splenectomy can be effective as salvage treatment even if relapsed thrombocytopenia occurs following PSE (14). Platelets have been found to increase from $5.4 \times 10^{4} / \mathrm{mm}^{3}$ prior to splenectomy to $18.4 \times 10^{4} / \mathrm{mm}^{3}$ thereafter (21), whereas following PSE, an increase from a mean value of $4.5 \times 10^{4}$ to $11.6 \times 10^{4} / \mathrm{mm}^{3}$ has been reported, although this was with infarcted splenic volume (12). Splenectomy is superior in terms of the level of the increase in platelet count and the low level of relapsed thrombocytopenia following platelet increase. For this reason, splenectomy is expected to be more reliable in raising platelet counts in cases of severe thrombocytopenia and it is highly likely that this increase may be sufficient for IFN therapy.

The results of IFN therapy in the present study are discussed below. As shown in Table I, IFN could not be initiated prior to splenectomy. IFN therapy was initiated in all the patients following splenectomy. SVR was not observed in patients with genotype 1 , however, it was observed in three patients (75\%) with genotype 2 . Due to severe side effects, including interstitial pneumonitis, $\mathrm{HCV}$-associated glomerulonephritis or leukopenia, five patients with genotype 1 discontinued IFN therapy. All the patients with genotype 2 were able to continue IFN therapy. These data suggest that in patients with genotype 2 in particular, splenectomy did improve the effectiveness 
of IFN therapy. However, in subjects infected with genotype 1, the result was far less satisfactory. The presence of advanced fibrosis and cirrhosis has always presented an obstacle to obtaining viral eradication during antiviral therapy $(22,23)$. The results of therapy differ according to the different clinical stages of cirrhosis (24). As the severity of liver disease deteriorates, the rate of SVR decreases and viral eradication is often hampered by the occurrence of adverse events. The development or exacerbation of cytopenia in patients with cirrhosis may increase the risks of infection, bleeding, anemia-related fatigue and poor stamina or exercise intolerance, eventually causing the patient to withdraw from the treatment program. Furthermore, when HCV-related LC patients with portal hypertension were treated with the PEG-IFN + RBV cotherapy, 14 out of 51 patients had to cease treatment within 24 weeks due to adverse events (25). In the present study, numerous patients had severe fibrosis or portal hypertension, as is reflected in the platelet counts, and the figure for SVR was low, particularly in genotype 1 patients. The relatively poor SVR rate in patients with genotype 1 mirrors other studies (26). The rate of SVR with PEG-IFN + RBV ranged from 10 to $44 \%$ for HCV genotypes $1 / 4$ and 33 to $72 \%$ for HCV genotypes $2 / 3$ in compensated cirrhosis (26). This treatment is thus highly useful in genotype 2 patients.

As well as increased leukocyte and platelet counts, the present subjects also demonstrated long-term improvement in liver function. Improved liver function following splenectomy has been previously reported (16). In the present study, liver function was likely to improve 5 years following splenectomy. The reasons for the improvement in liver function following splenectomy included: i) the resolution of portal hypertension; ii) the removal of hepatic regeneration inhibition factors in the spleen; iii) the alleviation of the prehepatic bilirubin burden due to a decrease in sites of erythrocyte destruction and iv) the enhancement of hepatic regeneration promotion factors. This discussion focuses on the removal of hepatic regeneration inhibition factors and the enhancement of hepatic regeneration promotion factors. Several studies have focused on the removal of hepatic regeneration inhibition factors. For example, in LC rats, TGF- $\beta$ derived from the spleen and hepatic stellate cells inhibited liver regeneration and splenectomy improved liver regeneration and prevented the progression of liver fibrosis $(27,28)$. In LC with portal hypertension, hepatic stellate cells are activated (29) and TGF- $\beta$ is overproduced as a result of splenic cell activation (27). It is likely that splenectomy improves liver regeneration due to loss of the TGF- $\beta$ signal from the spleen and the downregulation of TGF- $\beta$ from hepatic stellate cells due to the alleviation of portal hypertension.

Focus has been given to liver regeneration factors, including evidence that splenectomy leads to enhanced liver regeneration via the liver regeneration initiator TNF- $\alpha$ (30). Studies have demonstrated that platelets have marked effects in promoting liver regeneration (31). A review (32) presented experimental evidence demonstrating that platelets accelerate liver regeneration, involving three different mechanisms: i) the direct effect on hepatocytes, where platelets translocate to the space of Disse and release growth factors through direct contact with hepatocytes; ii) the co-operative effect with liver sinusoidal endothelial cells, where the dense concentration of sphingosine-1-phosphate in platelets induces excretion of interleukin- 6 from liver sinusoidal endothelial cells and iii) the collaborative effect with Kupffer cells, where the functions of Kupffer cells are enhanced by platelets.

Carcinogenesis was also rare in patients with previous HCC who did not achieve SVR. This was possibly due to two mechanisms, one of which is the cancer inhibition effect due to the reduction of portal pressure. The other mechanism was due to the use of IFN therapy. Although the extent of the decrease in portal pressure following splenectomy was not measured in the present study, previous studies have reported lower portal pressure following splenectomy. Ripoll et al demonstrated that the hepatic venous pressure gradient predicts the development of HCC independently of the severity of cirrhosis (33). It is thus possible that decreased portal pressure and decreased carcinogenesis are involved.

The use of IFN therapy, including long-term IFN monotherapy, also appears to have benefits in patients not achieving SVR. For example, the hepatitis $\mathrm{C}$ antiviral long-term treatment against cirrhosis (HALT-C) trial was designed to confirm the effect of PEG-IFN half-dose administration to patients who did not achieve SVR in previous IFN therapy. Extended analysis of the HALT-C cohort demonstrated that patients with cirrhosis who received PEG-IFN treatment had a reduced incidence of $\mathrm{HCC}$ (34).

The effect on blood biochemistry persisted over the long term. The long-term persistence of this effect was possibly due to splenectomy as well as antiviral therapy. In SVR patients and those with no virological response, very few patients with no previous history of HCC developed HCC and all achieved long-term survival. Although recurrence was not suppressed in patients with previous HCC, liver function was maintained and repeat treatment was possible. The present was a retrospective study, however, despite its limitations, further development of these treatments is anticipated. In HCV-related LC with severe thrombocytopenia, splenectomy and subsequent IFN therapy is potentially useful in improving the inhibition of fibrosis, suppressing $\mathrm{HCC}$ and improving survival rates. However, future investigations using new antiviral therapy, including protease inhibitors are crucial in order to overcome the low rate of SVR in genotype 1 patients.

\section{References}

1. Yoshida H, Shiratori Y, Moriyama M, et al: Interferon therapy reduces the risk for hepatocellular carcinoma: national surveillance program of cirrhotic and noncirrhotic patients with chronic hepatitis C in Japan. Ann Intern Med 131: 174-181, 1999.

2. Bruno S, Stroffolini T, Colombo M, et al: Sustained virological response to interferon- $\alpha$ is associated with improved outcome in HCV-related cirrhosis: a retrospective study. Hepatology 45: 579-587, 2007.

3. Ikeda K, Saitoh S, Arase Y, et al: Effect of interferon therapy on hepatocellular carcinogenesis in patients with chronic hepatitis C: A long-term observation study of 1,643 patients using statistical bias correction with proportional hazard analysis. Hepatology 29: 1124-1130, 1999

4. Tanaka H, Tsukuma H, Kasahara A, et al: Effect of interferon therapy on the incidence of hepatocellular carcinoma and mortality of patients with chronic hepatitis C: a retrospective cohort study of 738 patients. Int J Cancer 87: 741-749, 2000.

5. Poynard T, McHutchison J, Manns M, et al: Impact of pegylated interferon alfa-2b and ribavirin on liver fibrosis in patients with chronic hepatitis C. Gastroenterology 122: 1303-1313, 2002 . 
6. Ahmed F and Jacobson IM: Management of hematologic side effects: impact on compliance and efficacy. Cur Hepat Rep 4: 56-60, 2005.

7. Shiffman ML, Ghany MG, Morgan TR, et al: Impact of reducing peginterferon alfa-2a and ribavirin dose during retreatment in patients with chronic hepatitis C. Gastroenterology 132: 103-112, 2007.

8. McHutchson JG, Manns M, Patel K, et al: Adherence to combination therapy enhances sustained response in genotype-1-infected patients with chronic hepatitis C. Gastroenterology 123: 1061-1069, 2002.

9. Kercher KW, Carbonell AM, Heniford B, et al: Laparoscopic splenectomy reverses thrombocytopenia in patients with hepatitis C cirrhosis and portal hypertension. J Gastrointest Surg 8: 120-126, 2004.

10. Cai YQ, Zhou J, Chen XD, et al: Laparoscopic splenectomy is an effective and safe intervention for hypersplenism secondary to liver cirrhosis. Surg Endosc 25: 3791-3797, 2011

11. Murata K, Shiraki K, Takase K, et al: Long term follow-up for patients with liver cirrhosis after partial splenic embolization. Hepatogastroenterology 43: 1212-1217, 1996.

12. Hayashi H, Beppu T, Masuda T, et al: Predictive factors for platelet increase after partial splenic embolization in liver cirrhosis patients. J Gastroenterol Hepatol 22: 1638-1642, 2007.

13. McHutchison JG, Dusheiko G, Shiffman ML, et al: Eltrombopag for thrombocytopenia in patients with cirrhosis associated with hepatitis C. N Engl J Med 357: 2227-2236, 2007.

14. Hashimoto N, Akahoshi T, Tomikawa M, et al: Value of laparoscopic splenectomy as salvage treatment for relapsed thrombocytopenia after partial splenic arterial embolization. Dig Surg 27: 515-520, 2010.

15. Bisharat N, Omari H, Lavi I, et al: Risk of infection and death among post-splenectomy patients. J Infect 43: 182-186, 2001

16. Murata K, Ito K, Yoneda K, et al: Splenectomy improves liver function in patients with liver cirrhosis. Hepatogastroenterology 55: 1407-1411, 2008.

17. Morihara D, Kobayashi M, Ikeda K, et al: Effectiveness of combination therapy of splenectomy and long-term interferon in patients with hepatitis $C$ virus-related cirrhosis and thrombocytopenia. Hepatol Res 39: 439-447, 2009.

18. Ikezawa K, Naito M, Yumida T, et al: Splenectomy and antiviral treatment for thrombocytopenic with chronic hepatitis $\mathrm{C}$ virus infection. J Viral Hepat 17: 488-492, 2010.

19. Akahoshi T, Tomikawa M, Korenaga D, et al: Laparoscopic splenectomy with peginterferon and ribavirin therapy for patients with hepatitis C virus cirrhosis and hypersplenism. Surg Endosc 24: 680-685, 2010.
20. Tomikawa M, Akahoshi T, Sugimachi K, et al: Laparoscopic splenectomy may be a superior intervention for cirrhotic patients with hypersplenism. J Gastroenterol Hepatol 25: 397-402, 2010.

21. Yoshida D, Nagao Y, Tomikawa M, et al: Predictive factors for platelet count after laparoscopic splenectomy in cirrhotic patients. Hepatol Int: Sep 30, 2011 (Epub ahead of print).

22. Pawlotsky JM: Therapy of hepatitis C: from empiricism to eradication. Hepatology 43 (Suppl 1): S207-S220, 2006.

23. Zeuzem S: Heterogeneous virologic response rates to interferon based therapy in patients with chronic hepatitis C: who responds less well? Ann Intern Med 140: 370-381, 2004

24. Piccinino F and Coppola N: Antiviral treatment of HCV-related cirrhosis. Dig Liver Dis 39 (Suppl 1): S96-S101, 2007.

25. Di Marco V, Almasio PL, Ferraro D, et al: Peg-interferon alone or combined with ribavirin in HCV cirrhosis with portal hypertension: a randomized controlled trial. J Hepatol 47: 484-491, 2007.

26. Vezali E, Aghemo A and Colombo M: A review of the treatment of chronic hepatitis $\mathrm{C}$ virus infection in cirrhosis. Clin Ther 32: 2117-2138, 2010.

27. Nakamura T, Sakata R, Ueno T, et al: Inhibition of transforming grow th factor $\beta$ prevents progression of liver fibrosis and enhances hepatocyte regeneration in dimethylnitrosamine-treated rats. Hepatology 32: 247-255, 2000

28. Akahoshi T, Hashizume M, Tanoue K, et al: Role of the spleen in liver fibrosis in rats may be mediated by transforming growth factor $\beta$-1. J Gastroenterol Hepatol 17: 59-65, 2002.

29. Okada Y, Tsuzuki Y, Hokari R, et al: Pressure loading and ethanol exposure differently modulate rat hepatic stellate cell activation. J Cell Physiol 215: 472-480, 2008

30. Murata K, Shiraki K, Sugimoto K, et al: Splenectomy enhances liver regeneration through tumor necrosis factor (TNF)- $\alpha$ following dimethylnitrosamine-induced cirrhotic rat model. Hepatogastroenterology 48: 1022-1027, 2001.

31. Murata S, Ohkohchi N, Matsuo R, et al. Platelets promote liver regeneration in early period after hepatectomy in mice. World J Surg 31: 808-816, 2007.

32. Takahashi K, Murata S, Ohkohchi N, et al: Novel therapy for liver regeneration by increasing the number of platelets. Surg Today: Nov 21, 2012 (Epub ahead of print).

33. Ripoll C, Groszmann RJ, Garcia-Tsao G, et al: Hepatic venous pressure gradient predicts development of hepatocellular carcinoma independently of severity of cirrhosis. J Hepatol 50: 923-928, 2009.

34. Lok AS, Everhart JE, Wright EC, et al: Maintenance peginterferon therapy and other factors associated with hepatocellular carcinoma in patients with advanced hepatitis C. Gastroenterology 140: 840-849, 2011. 